\title{
$\sigma$-WEAKLY CLOSED MODULES OF CERTAIN REFLEXIVE OPERATOR ALGEBRAS
}

\author{
CHEN PEIXIN
}

(Communicated by Palle E. T. Jorgensen)

\begin{abstract}
Let $\mathscr{A}$ be a completely distributive CSL algebra and let $M$ be any $\sigma$-weakly closed $\mathscr{A}$-module. We give characterizations of commutant $C(\mathscr{A}, M)$ of $\mathscr{A}$ modulo $M$ and $\operatorname{AlgLat} M$. Furthermore, we deal with the relations among $\mathscr{A}, C(\mathscr{A}, M)$ and AlgLat $M$.
\end{abstract}

\section{Preliminaries and notation}

Throughout this paper, $H$ will denote a complex Hilbert space, and $L(H)$ will denote the Banach algebra of all bounded linear operators from $H$ into itself. Let $\mathscr{L}$ be a commutative and completely distributive subspace lattice on $H$ and let $\mathscr{A}=\operatorname{Alg} \mathscr{L} \subseteq L(H)$. A subspace $\mathscr{M}$ of $L(H)$ is said to be a $\mathscr{A}$-module if $\mathscr{A} \mathscr{M}=\mathscr{M} \mathscr{A} \subseteq \mathscr{M}$. Any $\sigma$-weakly closed $\mathscr{A}$-module is denoted by $M$ and the set of all $V$-generators of $\mathscr{L}$ is denoted by $\mathscr{G}$. The terminology and notation of this paper concerning reflexive operator algebras may be found in [1, 3, 4]. It is known from [3] that $M$ has the form

$$
M=\{T \in L(H): T E \subseteq \widetilde{E} \text { for all } E \in \mathscr{L}\}
$$

where $E \mid \rightarrow \widetilde{E}$ is some lattice homomorphism of $\mathscr{L}$ into itself. Thus we need only consider $M$ determined by the homomorphism $E \mid \rightarrow \widetilde{E}$.

\section{Commutants Relative to $\sigma$-Weakly Closed $\mathscr{A}$-MOdUles}

In [1] the authors found that $C(\operatorname{Alg} \mathscr{N}, M)=\mathscr{C}_{M} \oplus M$ for a nest $\mathscr{N}$, where $\mathscr{C}_{M}$ is a weakly closed subspace (is also a subalgebra) of the core of $\operatorname{Alg} \mathscr{N}$. A natural question is: Does this hold for $\mathscr{L}$ ? In this case when $M \supseteq \mathscr{A}$ Han Deguang proved $C(\mathscr{A}, M)=M$ [3]. Generally, we obtain $C(\mathscr{A}, M)=\mathscr{C}_{M}(G) \oplus M$, where $\mathscr{C}_{M}(G)$ is a weakly closed subspace of the core of $\mathscr{A}$.

We omit the proof of Lemma 2.1 as it follows by modification of the arguments in [4, p. 505].

Received by the editors June 25, 1993 and, in revised form, September 23, 1993.

1991 Mathematics Subject Classification. Primary 47D25, 47D15; Secondary 47B47.

Key words and phrases. $\mathscr{A}$-module, reflexive operator algebra, commutant of $\mathscr{A}$ modulo module. 
Lemma 2.1. If $T \in \mathscr{A}^{\prime}$, then each $\vee$-generator of $\mathscr{L}$ is contained in an eigenspace corresponding to the eigenvalue $\lambda$ of $T$ and $\|\lambda\| \leq\|T\|$.

Lemma 2.2. If $E \in \mathscr{L}$ and $T \in C(\mathscr{A}, M)$, then

(i) $(I-\widetilde{E}) T E G=\lambda_{T E}(G)(E-\widetilde{E} E) G$ and $\left|\lambda_{T E}(G)\right| \leq\|T\|$, where $G$ is any $V$-generator of $\mathscr{L}$ and $\lambda_{T E}(G)$ is a number depending on $T, E$, and $G$;

(ii) if $\widetilde{E} E \leq \widetilde{F} F<E<F$

$$
\lambda_{T E}(G)=\lambda_{T F}(G)
$$

where $F \in \mathscr{L}$, and $\lambda_{T E}(G)$ and $\lambda_{T F}(G)$ are as in (i).

Proof. (i) Since $E \in \mathscr{A}, I-\widetilde{E} \in \mathscr{A}$, and $T \in C(\mathscr{A}, M)$

$$
(I-\widetilde{E})(T E-E T) E=0, \quad(I-\widetilde{E})[T(I-\widetilde{E})-(I-\widetilde{E}) T] E=0 .
$$

Therefore

$$
(I-\widetilde{E}) T(E-\widetilde{E} E)=(I-\widetilde{E}) T E=(E-\widetilde{E} E) T E
$$

and hence

Now for any $A \in \mathscr{A}$

$$
(I-\widetilde{E}) T E=(E-\widetilde{E} E) T(E-\widetilde{E} E) .
$$

$$
(I-\widetilde{E})(T A-A T) E=0
$$

and since $(E-\widetilde{E} E) A(E-\widetilde{E} E) \in \mathscr{A}$

$$
\begin{aligned}
& (I-\widetilde{E}) T E[(E-\widetilde{E} E) A(E-\widetilde{E} E)]-[(E-\widetilde{E} E) A(E-\widetilde{E} E)](I-\widetilde{E}) T E \\
& \quad=(I-\widetilde{E})[T(E-\widetilde{E} E) A(E-\widetilde{E} E)-(E-\widetilde{E} E) A(E-\widetilde{E} E) T] E=0 .
\end{aligned}
$$

This means that $(I-\widetilde{E}) T E=(E-\widetilde{E} E) T(E-\widetilde{E} E) \in[(E-\widetilde{E} E) \mathscr{A}(E-\widetilde{E} E)]^{\prime}$. Apply Lemma 2.1 to the compression of $\mathscr{A}$ to the range of $E-\widetilde{E} E$

$$
\begin{aligned}
&(I-\widetilde{E}) T E G=[(E-\widetilde{E} E) T(E-\widetilde{E} E)][(E-\widetilde{E} E) G(E-\widetilde{E} E)] \\
&=\lambda_{T E}(G)[(E-\widetilde{E} E) G(E-\widetilde{E} E)] \\
&=\lambda_{T E}(G)(E-\widetilde{E} E) G \\
&\left|\lambda_{T E}(G)\right| \leq\|(E-\widetilde{E} E) T(E-\widetilde{E} E)\| \leq\|T\| .
\end{aligned}
$$

(ii) Observe

$$
\begin{aligned}
(E-\widetilde{E} E) T(E-\widetilde{E} E) G & =\lambda_{T E}(G)(E-\widetilde{E} E) G, \\
(F-\widetilde{F} F) T(F-\widetilde{F} F) G & =\lambda_{T F}(G)(F-\widetilde{F} F) G .
\end{aligned}
$$

Multiplying the above on either side by $E-\widetilde{F} F$, we obtain

$$
\begin{aligned}
(E-\widetilde{F} F) T(E-\widetilde{F} F) G & =\lambda_{T E}(G)(E-\widetilde{F} F) G, \\
(E-\widetilde{F} F) T(E-\widetilde{F} F) G & =\lambda_{T F}(G)(E-\widetilde{F} F) G .
\end{aligned}
$$

Thus

$$
\lambda_{T E}(G)=\lambda_{T F}(G)
$$

and (ii) follows.

Remark 2.3. Lemma 2.2 shows that for $T \in C(\mathscr{A}, M)$ and $G \in \mathscr{G}, \lambda_{T E}(G)$ has a constant value on the intervals $\left(\widetilde{E}_{1} E_{1}, E_{2}\right]$ and $\left(\widetilde{E}_{2} E_{2}, E_{1}\right]$ if intervals $\left(\widetilde{E}_{1} E_{1}, E_{1}\right)$ and $\left(\widetilde{E}_{2} E_{2}, E_{2}\right)$ overlap. It follows easily that $\lambda_{T E}(G)$ is constant on each maximal connected component of $\bigcup\{(\widetilde{E} E, E]: E \in \mathscr{L}\}$. 
Definition. The element $F$ of $\mathscr{L}$ is $\sim$ connected to $E$ (notation $E \lesssim F$ or $F \gtrsim E)$ if $E=F$ or $E<F$ and exists a finite chain $E_{n}<\cdots<E_{1}<E_{0}=F$ with $\widetilde{E}_{i} E_{i}<E_{i+1} \quad(0 \leq i \leq n-1)$ and $\widetilde{E}_{n} E<E$.

Let $\mathscr{L}_{0}=\{E \in \mathscr{L}: \widetilde{E} E<E\}$. For each $E \in \mathscr{L}_{0}$ we define the -component $\gamma(E)$ of $E$ by

$$
\gamma(E)=\{F \in \mathscr{L}: F \lesssim E\} \cup\{F \in \mathscr{L}: F \gtrsim E\} .
$$

Clearly, $\mathscr{L}_{0}$ is a disjoint union of $\sim$-components and it is easy to see that $\sim$-components are intervals. We may write

$$
\mathscr{L}_{0}=\bigcup_{\omega \in \Omega} \gamma \omega
$$

where $\Omega$ is some index set and $\left\{\gamma_{\omega}: \omega \in \Omega\right\}$ are pairwise disjoint intervals with left end point $E_{\omega}$ and right end point $F_{\omega}$.

For fixed $T \in C(\mathscr{A}, M)$ and $F \in \mathscr{L}$, it is possible that $G_{1}, G_{2} \in \mathscr{G}$ and $G_{1} \neq G_{2}$ but $\lambda_{T F}\left(G_{1}\right)=\lambda_{T F}\left(G_{2}\right)$. Let $G_{T F}(\alpha)$ be the closed linear span of all $\vee$-generators of $\mathscr{L}$ corresponding to the same eigenvalue $\lambda_{T F}(\alpha)$ of $(I-\widetilde{F}) T F$, where $\alpha \in \Lambda$ and $\Lambda$ is some index set. Lemma 2.2 shows that

$$
G_{T E}(\alpha)=G_{T F}(\alpha)
$$

for all $E, F \in \gamma_{\omega}$; we denote it by $G_{T \omega}(\alpha)$. Clearly, $G_{T \omega}(\alpha) \in \mathscr{L}$ and $I=\bigvee_{\alpha \in A} G_{T \omega}(\alpha)$. If $T \in C(\mathscr{A}, M), \omega \in \Omega$ are given and $E \in \gamma_{\omega}$, then

$$
\begin{gathered}
(E-\tilde{E} E) G_{T \omega}(\alpha) G_{T \omega}(\beta)=G_{T \omega}(\alpha) G_{T \omega}(\beta)(E-\widetilde{E} E) \\
\quad= \begin{cases}(E-\widetilde{E} E) G_{T \omega}(\alpha) & \text { if } \alpha=\beta, \\
0 & \text { if } \alpha \neq \beta .\end{cases}
\end{gathered}
$$

Denote by $\mathscr{C}_{M}(G)$ the weakly closed linear span generated by the projections $\left\{\left(F_{\omega}-E_{\omega}\right) G_{T \omega}(\alpha): T \in C(\mathscr{A}, M), \omega \in \Omega\right.$, and $\left.\alpha \in \Lambda\right\}$.

Theorem 2.4. $C(\mathscr{A}, M)=\mathscr{C}_{M}(G) \oplus M$, where the sum is a direct sum of vector spaces.

Proof. Suppose $T \in C(\mathscr{A}, M), \omega \in \Omega$, and $\alpha \in \Lambda$ are given. For any $E \in \mathscr{L}$, there is a $\sim$-component $\gamma_{\omega_{0}}$ of $\mathscr{L}_{0}$ such that $E \in \gamma_{\omega_{0}}$. For any $A \in \mathscr{A}$, if $\omega_{0} \neq \omega$,

$$
(I-\widetilde{E})\left[A\left(F_{\omega}-E_{\omega}\right) G_{T \omega}(\alpha)-\left(F_{\omega}-E_{\omega}\right) G_{T \omega}(\alpha) A\right] E=0 ;
$$

if $\omega_{0}=\omega$, then for any $\beta \in \Lambda$

$$
\begin{aligned}
(E-\widetilde{E} E)\left[A G_{T \omega}(\alpha)-G_{T \omega}(\alpha) A\right](E-\widetilde{E} E) G_{T \omega}(\beta) \\
=(E-\widetilde{E} E) G_{T \omega}(\alpha) G_{T \omega}(\beta) A(E-\widetilde{E} E) G_{T \omega}(\alpha) G_{T \omega}(\beta) \\
\quad-(E-\widetilde{E} E) G_{T \omega}(\alpha) G_{T \omega}(\beta) A(E-\widetilde{E} E) G_{T \omega}(\beta) \\
=0 .
\end{aligned}
$$


Hence

$$
\begin{aligned}
(I- & \widetilde{E})\left[A\left(F_{\omega}-E_{\omega}\right) G_{T \omega}(\alpha)-\left(F_{\omega}-E_{\omega}\right) G_{T \omega}(\alpha) A\right] E \\
& =(I-\widetilde{E}) A\left[(I-\widetilde{E})\left(F_{\omega}-E_{\omega}\right) E\right] G_{T \omega}(\alpha)-\left[(I-\widetilde{E})\left(F_{\omega}-E_{\omega}\right) E\right] G_{T \omega}(\alpha) A E \\
& =(E-\widetilde{E} E)\left[A G_{T \omega}(\alpha)-G_{T \omega}(\alpha) A\right](E-\widetilde{E} E) \\
& =(E-\widetilde{E} E)\left[A G_{T \omega}(\alpha)-G_{T \omega}(\alpha) A\right](E-\widetilde{E} E)\left(\bigvee_{\beta \in \Lambda} G_{T \omega}(\beta)\right) \\
& =0,
\end{aligned}
$$

so for all $S \in \mathscr{C}_{M}(G)$

$$
(I-\widetilde{E})(A S-S A) E=0 .
$$

This shows that $S \in C(\mathscr{A}, M)$, and thus the inclusion $\mathscr{C}_{M}(G)+M \subseteq C(\mathscr{A}, M)$ follows.

Now suppose $T \in C(\mathscr{A}, M)$; then it follows from Lemma 2.2 that if $\gamma_{\omega_{0}}$ is any $\sim$-component of $\mathscr{L}_{0}$ and $E \in \gamma_{\omega_{0}}$, we have

$$
(I-\tilde{E}) T E G_{T \omega_{0}}(\alpha)=\lambda_{T \omega_{0}}(\alpha)(E-\tilde{E} E) G_{T \omega_{0}}(\alpha)
$$

where $\lambda_{T \omega_{0}}(\alpha)$ is a number depending on $T, \gamma_{\omega_{0}}$, and $\alpha$. Define $T_{M}$ by

$$
T_{M}=\sum_{\omega \in \Omega} \sum_{\alpha \in A} \lambda_{T \omega}(\alpha)\left(F_{\omega}-F_{\omega}\right) G_{T \omega}(\alpha) \text {. }
$$

By Lemma 2.2, $\left|\lambda_{T \omega}(\alpha)\right| \leq\|T\|$; hence, the series converges in the strong operator topology and $T_{M} \in \mathscr{C}_{M}(G)$.

For any $\beta \in \Lambda$

$$
\begin{aligned}
& (I-\widetilde{E})\left(T-T_{M}\right) E G_{T \omega_{0}}(\beta)=(I-\widetilde{E}) T E G_{T \omega_{0}}(\beta)-(I-\widetilde{E}) T_{M} E G_{T \omega_{0}}(\beta) \\
& \quad=\lambda_{T \omega_{0}}(\beta)(E-\widetilde{E} E) G_{T \omega_{0}}(\beta)-\lambda_{T \omega_{0}}(\beta)(E-\widetilde{E} E) G_{T \omega_{0}}(\beta) \\
& \quad=0 .
\end{aligned}
$$

Hence

$$
(I-\widetilde{E})\left(T-T_{M}\right) E=(I-\widetilde{E})\left(T-T_{M}\right) E\left(\bigvee_{\beta \in \Lambda} G_{T \omega_{0}}(\beta)\right)=0
$$

and $T-T_{M} \in M$. Therefore $T \in \mathscr{C}_{M}(G)+M$.

To prove that the sum is direct, if $T \in \mathscr{C}_{M}(G)$, then for each $\omega \in \Omega$ and any $\beta \in \Lambda$

$$
T\left(F_{\omega}-E_{\omega}\right) G_{T \omega}(\beta)=\lambda_{T \omega}(\beta)\left(F_{\omega}-E_{\omega}\right) G_{T \omega}(\beta) .
$$

If also $T \in M$, choose $E \in \gamma_{\omega}$

$$
O=(I-\widetilde{E}) T E G_{T \omega}(\beta)=\lambda_{T \omega}(\beta)(E-\widetilde{E} E) G_{T \omega}(\beta) .
$$

Thus $\lambda_{T \omega}(\beta)=0$ for each $\omega \in \Omega$ and each $\beta \in \Lambda$, and

$$
T\left(F_{\omega}-E_{\omega}\right)=T\left(F_{\omega}-E_{\omega}\right)\left(\bigvee_{\beta \in \Lambda} G_{T \omega}(\beta)\right)=0 \text {. }
$$

Therefore $T=0$. The proof is completed. 


\section{THE OPERATOR Algebra Generated by A MODUle}

In this section, first we prove that AlgLat $M=\mathscr{C}_{M} \oplus\langle M\rangle$, where $\langle M\rangle$ is the weakly closed algebra generated by $M$. Furthermore, we deal with the relations among AlgLat $M, C(\mathscr{A},\langle M\rangle)$, and $\mathscr{A}$.

Lemma 3.1 is the analogue of Lemma 1.10 in [1], thus we omit the proof.

Lemma 3.1. The weakly closed algebra generated by $M$ is the module determined by $E \mid \rightarrow \widehat{E}$, where

$$
\widehat{E}= \begin{cases}\widetilde{E} & \text { if } \widetilde{E} \leq E, \\ \bigvee\left\{\widetilde{E}^{(n)}: n \geq 0\right\} & \text { if } \widetilde{E} \not E .\end{cases}
$$

Lemma 3.2 [3]. $P \in$ Lat $M$ if and only if there exists some $E \in \mathscr{L}$ such that $\widetilde{E} \leq P \leq E$.

Theorem 3.3. AlgLat $M=\mathscr{C}_{M} \oplus\langle M\rangle$, where $\mathscr{C}_{M}$ is the weakly closed algebra generated by $\left\{F_{\omega}-E_{\omega}: \omega \in \Omega_{0}\right\} . F_{\omega}$ and $E_{\omega}$ are the end points of the components of $\{E \in \mathscr{L}: \widetilde{E}<E\}, \Omega_{0} \subseteq \Omega$, and $\langle M\rangle$ is the weakly closed algebra generated by $M$.

Proof. Clearly AlgLat $M=\operatorname{AlgLat}\langle M\rangle$. Since $\mathscr{C}_{M}$ depends only on the elements $E$ of $\mathscr{L}$ such that $\widetilde{E}<E$, Lemma 3.1 shows that $\mathscr{C}_{M}=\mathscr{C}_{\langle M\rangle}$. Thus we need only prove the equation

$$
\operatorname{AlgLat}\langle M\rangle=\mathscr{C}_{\langle M\rangle} \oplus\langle M\rangle
$$

That AlgLat $\langle M\rangle \supseteq \mathscr{C}_{\langle M\rangle}+\langle M\rangle$ is obvious. Let $T \in \operatorname{AlgLat}\langle M\rangle$. From Lemma 3.2 for all $E \in \mathscr{L}$ with $\widetilde{E} \leq E$ and any $G \leq E-\widetilde{E}, \widetilde{E} \oplus G \in \operatorname{Lat} T$. Thus $(E-\widetilde{E}) T(E-\widetilde{E})$ leaves every subprojection of $E-\widetilde{E}$ invariant. This means that

$$
(E-\widetilde{E}) T(E-\widetilde{E})=\lambda_{T E}(E-\widetilde{E})
$$

for some scalar $\lambda_{T E}$. Note that $E \in \operatorname{Lat}\langle M\rangle \subseteq$ Lat $T$; we have

$$
(I-\widetilde{E}) T E=(E-\widetilde{E}) T(E-\widetilde{E})=\lambda_{T E}(E-\widetilde{E}) .
$$

If $E$ and $F$ are in the same -component it follows as in Lemma 2.2 that $\lambda_{T E}=\lambda_{T F}$. The proof is now completed by modification of the arguments in Theorem 2.4.

Definition. $M$ is said to have property (*) if for any $T \in C(\mathscr{A}, M), E \in \mathscr{L}$, there is a number $\lambda_{T E}$ such that

$$
(E-\widetilde{E}) T(E-\widetilde{E})=\lambda_{T E}(E-\widetilde{E}) .
$$

The following facts are easily seen.

(i) $M \supseteq \mathscr{A}$ iff $\widetilde{E} \geq E$ for all $E \in \mathscr{L}$;

(ii) $M \subseteq \mathscr{A}$ iff $\widetilde{E} \leq E$ for all $E \in \mathscr{L}$;

(iii) $\mathscr{C}_{M}(G) \subseteq \mathscr{A}$.

We can prove the following results by the above facts and Theorems 2.4 and 3.3.

Corollary 3.4. Let $\langle M\rangle$ be the weakly closed algebra generated by $M$. Then

(i) AlgLat $M \subseteq C(\mathscr{A},\langle M\rangle)$;

(ii) if $M \supseteq \mathscr{A}$, then AlgLat $M=\langle M\rangle$ and $C(\mathscr{A}, M)=M$;

(iii) if $M \subseteq \mathscr{A}$, then AlgLat $M \subseteq C(\mathscr{A}, M) \subseteq \mathscr{A}$. 
Furthermore, AlgLat $M=C(\mathscr{A}, M)$ iff $M$ has property $(*) ; C(\mathscr{A}, M)=$ $\mathscr{A}$ iff $(E-\widetilde{E})\left(A_{1} A_{2}-A_{2} A_{1}\right)(E-\widetilde{E})=0$ for all $A_{1}, A_{2} \in \mathscr{A}$ and $E \in \mathscr{L}$.

\section{ACKNOWLEDGMENT}

I am grateful to vice professor Han Deguang for his help and the referee for many suggestions.

\section{REFERENCES}

1. J. A. Erdos and S. C. Power, Weakly closed ideals of nest algebras, J. Operator Theory 7 (1983), 219-235.

2. J. A. Erdos, Reflexivity for subspace maps and linear spaces of operators, Proc. London Math. Soc. 52 (1986), 582-600.

3. Han Deguang, On $\mathscr{A}$-submodules for reflexive operators, Proc. Amer. Math. Soc. 104 (1988), 1067-1070.

4. M. S. Lambrou, Approximants commutants and double commutants in normed algebras, J. London Math. Soc. (2) 25 (1982), 499-512.

Department of Mathematics and Physics, University of Petroleum, Dongying City, Shandong Province, 257062, Peoples Republic of China 